\title{
Excited State Dynamics of 7-Deazaguanosine and Guanosine 5'-Monophosphate
}

\author{
Sarah E. Krul, ${ }^{1, \dagger}$ Sean J. Hoehn, ${ }^{1, \dagger}$ Karl Feierabend, ${ }^{1,2}$ Carlos E. Crespo-Hernández*,1 \\ ${ }^{1}$ Department of Chemistry, Case Western Reserve University, Cleveland, Ohio 44106, USA \\ 2 Permanent address: Department of Chemistry, The College of Wooster, Wooster, Ohio 44691, \\ USA
}

* email of corresponding author: carlos.crespo@case.edu, ${ }^{\dagger}$ these authors contributed equally to this work

\section{ORCID}

Sarah E. Krul: https://orcid.org/0000-0003-4201-039X

Sean J. Hoehn: https://orcid.org/0000-0002-8282-7807

Karl Feierabend: https://orcid.org/0000-0002-4221-2584

Carlos E. Crespo-Hernández: https://orcid.org/0000-0002-3594-0890

\begin{abstract}
Minor structural modifications to the DNA and RNA nucleobases have a significant effect on their excited state dynamics and electronic relaxation pathways. In this study, the excited state dynamics of 7-deazaguanosine and guanosine 5'-monophosphate are investigated in aqueous and in a mixture of methanol and water using femtosecond broadband transient absorption spectroscopy following excitation at $267 \mathrm{~nm}$. The transient spectra are collected using photon densities that ensure no parasitic multiphoton-induced signal from solvated electrons. The data can be fit satisfactorily using a two- or three-component kinetic model. By analyzing the results from steady-state, time-resolved, computational calculations, and the methanol-water mixture, the following general relaxation mechanism is proposed for both molecules, $\mathrm{L}_{b} \rightarrow \mathrm{L}_{\mathrm{a}} \rightarrow{ }^{1} \pi \sigma^{*}$ (ICT) $\rightarrow \mathrm{S}_{0}$, where the ${ }^{1} \pi \sigma^{*}(\mathrm{ICT})$ stands for an intramolecular charge transfer excited singlet state with significant $\pi \sigma^{*}$ character. In general, longer lifetimes for internal conversion are obtained for 7deazaguanosine compared to guanosine $5^{\prime}$-monophosphate. Internal conversion of the ${ }^{1} \pi \sigma^{*}$ (ICT) state to the ground state occurs on a similar time scale of a few picoseconds in both molecules. Collectively, the results demonstrate that substitution of a single nitrogen for a methine $(\mathrm{C}-\mathrm{H})$ group at position seven of the guanine moiety stabilizes the ${ }^{1} \pi \pi^{*} \mathrm{~L}_{b}$ and $\mathrm{L}_{\mathrm{a}}$ states and alter the topology of their potential energy surfaces in such a way that the relaxation dynamics in 7deazaguanosine are slowed down compared to those in guanosine 5'-monophosphate but not for the internal conversion of ${ }^{1} \pi \sigma^{*}(\mathrm{ICT})$ state to the ground state.
\end{abstract}




\section{Introduction}

Modern time-resolved spectroscopic experiments have shown that the canonical DNA monomers dissipate the excess electronic energy arising from absorption of UV radiation primarily through internal conversion from the ${ }^{1} \pi \pi^{*}$ states to the ground state $\left(\mathrm{S}_{0}\right)$ within hundreds of femtoseconds. ${ }^{1-3}$ This rapid dissipation of excess electronic energy allows nucleobases to be largely photostable and uniquely placed as the building blocks of life on Earth. Vast experimental and computational evidence have been presented demonstrating that minor chemical modifications of the pyrimidine and purine core can significantly alter the electronic relaxation pathways by modifying the topology of their excited state potential energy surfaces. ${ }^{4-15}$ Depending on the specific site of modification on the core, access to specific conical intersections can be made less favorable, often resulting in higher yields of longer-lived excited states and a decrease in photostability.
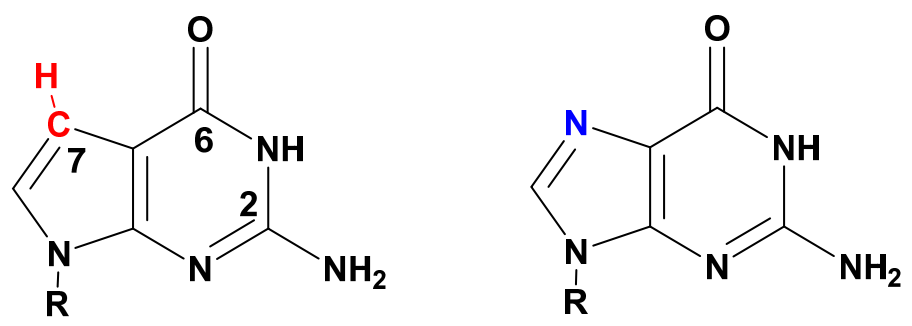

Scheme 1. Structures of 7-deazaguanosine (7dza) and guanosine 5'-monophosphate (GMP). R represents a 2'-deoxyribose group in 7dza and a ribose 5'-monophosphate group in GMP. The common nucleobase ring numbering is shown.

7-Deazaguanosine (7dza) differs in structure from guanosine solely by the replacement of a nitrogen atom with a $\mathrm{C}-\mathrm{H}$ group at the position seven of the guanine moiety (Scheme 1). This minor substitution results in ca. $25 \%$ reduction in the oxidation potential, from $1.24 \mathrm{~V}$ in guanosine to $0.95 \mathrm{~V}$ in $7 \mathrm{dza},{ }^{16-21}$ which has enabled the use of $7 \mathrm{dza}$ as a molecular probe to investigate charge transfer dynamics in DNA. ${ }^{20,22,23}$ To the best of our knowledge, however, the electronic relaxation 
mechanism of $7 \mathrm{dza}$ has not been investigated, precluding a direct comparison with the excitedstate dynamics of other purine monomers.

In this contribution, steady-state and time-resolved experiments are presented for $7 \mathrm{dza}$ in aqueous phosphate buffer solution at $\mathrm{pH} 6.8$ and in a mixture of $91 \%$ methanol and $9 \%$ water. The laser experimental conditions are optimized to eliminate the conventional requirement to correct the transient absorption data for the absorption of hydrated electrons formed from the two-photon ionization of the water solvent when excitation is performed at $267 \mathrm{~nm}$. In addition, newly collected broadband transient absorption experiments for GMP are compared with those collected for $7 \mathrm{dza}$ under equal experimental conditions. GMP is selected in this study for comparison with 7dza because its excited-state dynamics have been investigated in reasonable detail from both experimental and computational perspectives ${ }^{24-29}$ but also because of its biological relevance and greater solubility in aqueous solution than guanosine. The experimental results are also supplemented with ground and excited-state calculations at the density functional level of theory that take into consideration the dielectric constant of the solvent.

\section{Results}

\section{A. Steady-state absorption spectroscopy}

Figure 1 shows the steady-state absorptivity spectra of 7-deazaguanosine (7dza) and guanosine 5'-monophosphate (GMP) in aqueous phosphate buffer solution at $\mathrm{pH}$ 6.8. At this $\mathrm{pH}$, both molecules are in their keto tautomeric form. ${ }^{30} 7 \mathrm{dza}$ exhibits a band maximum at $259 \mathrm{~nm}$ and a shoulder at ca. $285 \mathrm{~nm}$, whereas GMP exhibits a band maximum at $252 \mathrm{~nm}$ and a shoulder at ca. $275 \mathrm{~nm}$. These results are consistent with those reported in literature for GMP, ${ }^{2,24,27}$ for the two lowest lying excited single $\pi \pi^{*}$ transitions, commonly referred to as the $\mathrm{Lb}_{b}\left(\mathrm{~S}_{2}\right)$ and $\mathrm{La}_{\mathrm{a}}\left(\mathrm{S}_{1}\right)$ states, 
respectively, using Platt's notation. ${ }^{31}$ Relative to GMP, 7dza absorption band maxima are redshifted by 7 and $10 \mathrm{~nm}$, respectively. Attempts were made to record steady-state fluorescence spectra for these molecules in aqueous solution but were unsuccessful due to the relative low sensitivity of the spectrometer available to us. Absorption and emission spectra were also collected in a mixture of $91 \%$ methanol and 9\% water (Figures S1 and S2). The absorption spectra are almost identical to those in aqueous solution for both molecules. Interestingly, however, while emission was within signal-to-noise in aqueous solution, low-intensity emission spectra were observed for both $7 \mathrm{dza}$ and GMP upon excitation at $267 \mathrm{~nm}$ in the mixture of $91 \%$ methanol and $9 \%$ water. Dual emission bands are observed for both 7dza and GMP with emission maxima around 330 and $415 \mathrm{~nm}$ for GMP, and around 310 and $430 \mathrm{~nm}$ for $7 \mathrm{dza}$ (Figure S2). We remark that these emission signals are within the sensitivity of the spectrometer used and the emission bands are broad.

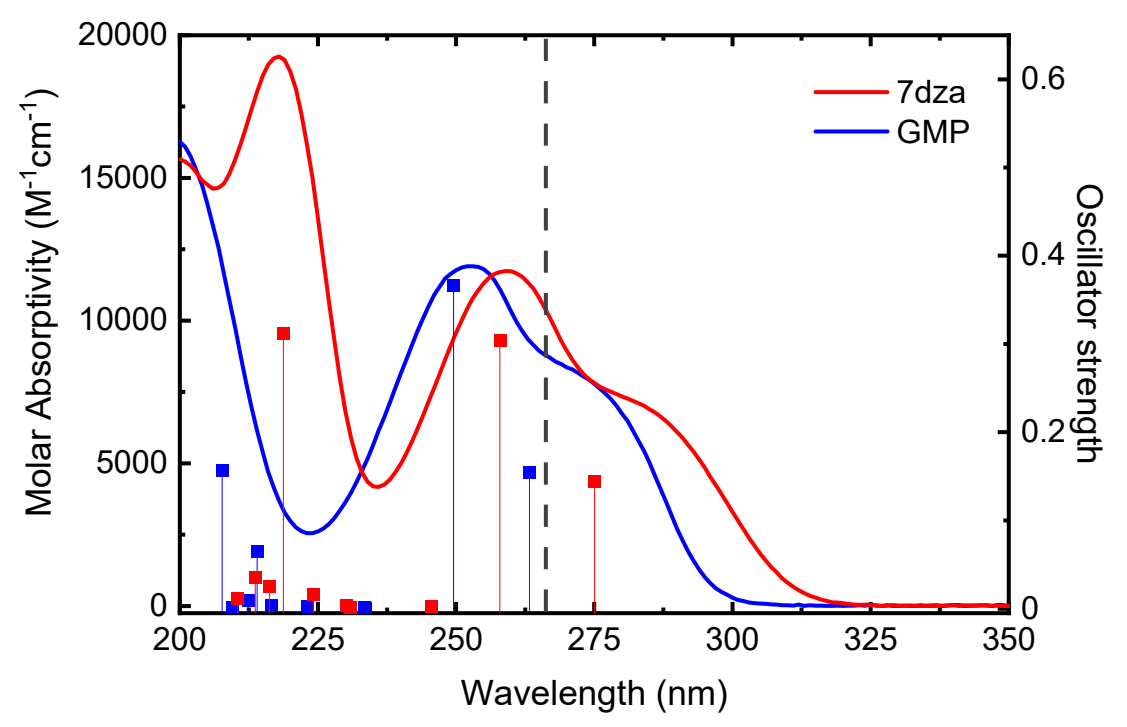

Figure 1. Ground-state absorptivity spectra of 7dza (red) and GMP (blue) in phosphate buffer aqueous solution at $\mathrm{pH}$ 6.8. Vertical lines represent the time-dependent density functional theory (TD-DFT) calculated vertical excitation energies for 7dza and GMP in the syn-sugar conformations using water as the solvent and the associated oscillator strengths at the TDPBE0/IEFPCM/6-311+G(d,p)//B3LYP/IEFPCM/6-311+G(d,p) level of theory. The dashed line indicates the excitation wavelength of $267 \mathrm{~nm}$ used in the laser experiments. 


\section{B. Quantum chemical calculations}

The ground state geometries of both 7dza and GMP were optimized at the B3LYP/IEFPCM/6-311+G(d,p) level of theory in water, followed by calculation of the vertical excitation energies at the TD-PBE0/IEFPCM/6-311+G(d,p) level of theory. Both syn and antisugar conformations were optimized for both molecules. The syn-sugar conformation is the most stable in both 7dza and GMP in water, while the anti-conformation resides 1.4 and $3.4 \mathrm{kcal} / \mathrm{mol}$ higher in energy, respectively, at the TD-PBE0/IEFPCM/6-311+G(d,p) level of theory (see Tables $\mathrm{S} 1$ and S2). The purine chromophore of both optimized structures is planar, while the amino group at the $\mathrm{C} 2$ is pyrimidalized in both molecules.

The energies and characters of the lowest four excited singlet and three triplet states for each molecule for the syn-sugar conformations are listed in Table 1. The percent contributions of the single electron transitions for each excited state are tabulated in Tables S3 and S4 for the synsugar conformations, whereas Tables S5 and S6 collect the energies and characters of the lowest two singlet and three triplet states and percent contributions of the single electron transitions for each excited state for the anti-sugar conformations. The Kohn-Sham orbitals are reported in Figures S3 and S4 for the syn-sugar conformations and in Figures S5 and S6 for the anti-sugar conformations.

The two lowest energy excited singlet states have $\pi \pi^{*}$ character for both $7 \mathrm{dza}$ and GMP. These states are slightly red shifted by approximately $0.2 \mathrm{eV}$ for $7 \mathrm{dza}$ relative to GMP, which corresponds well with the red shift in steady-state absorption spectra shown in Figure 1. The third excited singlet state for both molecules has relatively small oscillator strength (ca. $10^{-3}$ ) and the primary single-electron transitions exhibit significant charge transfer from the $\pi$-orbitals of the purine chromophore to the sugar-phosphate and/or the amino groups (see KS orbitals in Figures 
$\mathrm{S} 3$ to S6), while the fourth excited singlet state has primarily $n \pi *$ character. The equal ordering and character of electronic states for 7dza and GMP in water suggests that $\mathrm{N}$-for-CH substitution at the position seven of the purine chromophore does not affect the order of the lowest energy excited singlet states appreciably in the Franck-Condon region. It is important to note that both the $\mathrm{L}_{\mathrm{a}}$ and $\mathrm{L}_{\mathrm{b}}$ singlet excited states of 7dza and GMP can be simultaneously populated upon excitation at $267 \mathrm{~nm}$ in aqueous solution, while, within the expected accuracy of the calculations of \pm 0.3 $\mathrm{eV},{ }^{32}$ the third excited singlet state may also be within reach upon excitation at $267 \mathrm{~nm}$. According to the KS orbitals, the third singlet excited state for both 7dza and GMP is an intramolecular charge transfer (ICT) state with sizable $\pi \sigma^{*}$ character. Similar ICT states have been previously observed by Tuna and Domcke for adenosine ${ }^{33}$ and 8 -oxo-deoxyguanosine. ${ }^{34}$

Table 1. Vertical excitation energies (in $\mathrm{eV}$ ) for the lowest four excited singlet states and three excited triplet states of the syn-sugar conformation of 7-deazaguanosine (7dza) and guanosine 5'monophosphate (GMP) in water calculated at the TD-PBE0/IEFPCM/6$311+\mathrm{G}(\mathrm{d}, \mathrm{p}) / / \mathrm{B} 3 \mathrm{LYP} / \mathrm{IEFPCM} / 6-311+\mathrm{G}(\mathrm{d}, \mathrm{p})$ level of theory. The respective oscillator strengths are listed in parentheses.

\begin{tabular}{lll}
\hline State & 7dza & GMP \\
\hline $\mathrm{S}_{1}\left(\pi \pi^{*}, \mathrm{~L}_{\mathrm{a}}\right)$ & $4.7(0.1448)$ & $4.9(0.1541)$ \\
$\mathrm{S}_{2}\left(\pi \pi^{*}, \mathrm{~L}_{\mathrm{b}}\right)$ & $5.0(0.3044)$ & $5.2(0.3668)$ \\
$\mathrm{S}_{3}\left(\pi \sigma^{*}, \mathrm{ICT}\right)$ & $5.2(0.0026)$ & $5.5(0.0021)$ \\
$\mathrm{S}_{4}\left(\mathrm{n} \pi^{*}\right)$ & $5.6(0.0019)$ & $5.5(0.0004)$ \\
$\mathrm{T}_{1}\left(\pi \pi^{*}\right)$ & 3.6 & 3.6 \\
$\mathrm{~T}_{2}\left(\pi \pi^{*}\right)$ & 3.6 & 3.9 \\
$\mathrm{~T}_{3}\left(\pi \pi^{*}\right)$ & 4.6 & 4.8
\end{tabular}




\section{Femtosecond broadband transient absorption spectroscopy}

Figure 2 shows the transient absorption spectra for 7dza (a-b) and GMP (c-d) following excitation at $267 \mathrm{~nm}$ in aqueous buffer solution at $\mathrm{pH}$ 6.8. One-photon excitation conditions were used to avoid the two-photon ionization of the water solvent and, therefore, the need for correction of the hydrated electron absorption band (see Methods for details). Excitation of 7dza results in the observation of transient spectra with a band maximum below $330 \mathrm{~nm}$ and a simultaneous band with absorption maximum around $550 \mathrm{~nm}$ that blue shifts to ca. $480 \mathrm{~nm}$ within the cross-correlation of the pump and probe beams (Figure 2a). Subsequently, the $480 \mathrm{~nm}$ band decays in a few picoseconds, while the band with absorption maximum below $330 \mathrm{~nm}$ decays within ca. $11 \mathrm{ps,}$ after which point no further transient absorption signal is detected (Figure $2 \mathrm{~b}$ ). Figure 2c shows the transient absorption spectra of GMP within the cross-correlation of the pump and probe beams. The absorption spectrum at time zero has maxima below $330 \mathrm{~nm}$, at ca. 425 , and $575 \mathrm{~nm}$. The transient absorption spectra fully decay within ca. 10 ps. However, as shown in Figure $2 \mathrm{~d}$ and Figure $\mathrm{S} 7 \mathrm{~b}$, the band with a maximum around $575 \mathrm{~nm}$ decays faster than the absorption band at ca. 330 , while the band at $475 \mathrm{~nm}$ seems to decay as a linear combination of the 330 and $575 \mathrm{~nm}$ bands. 

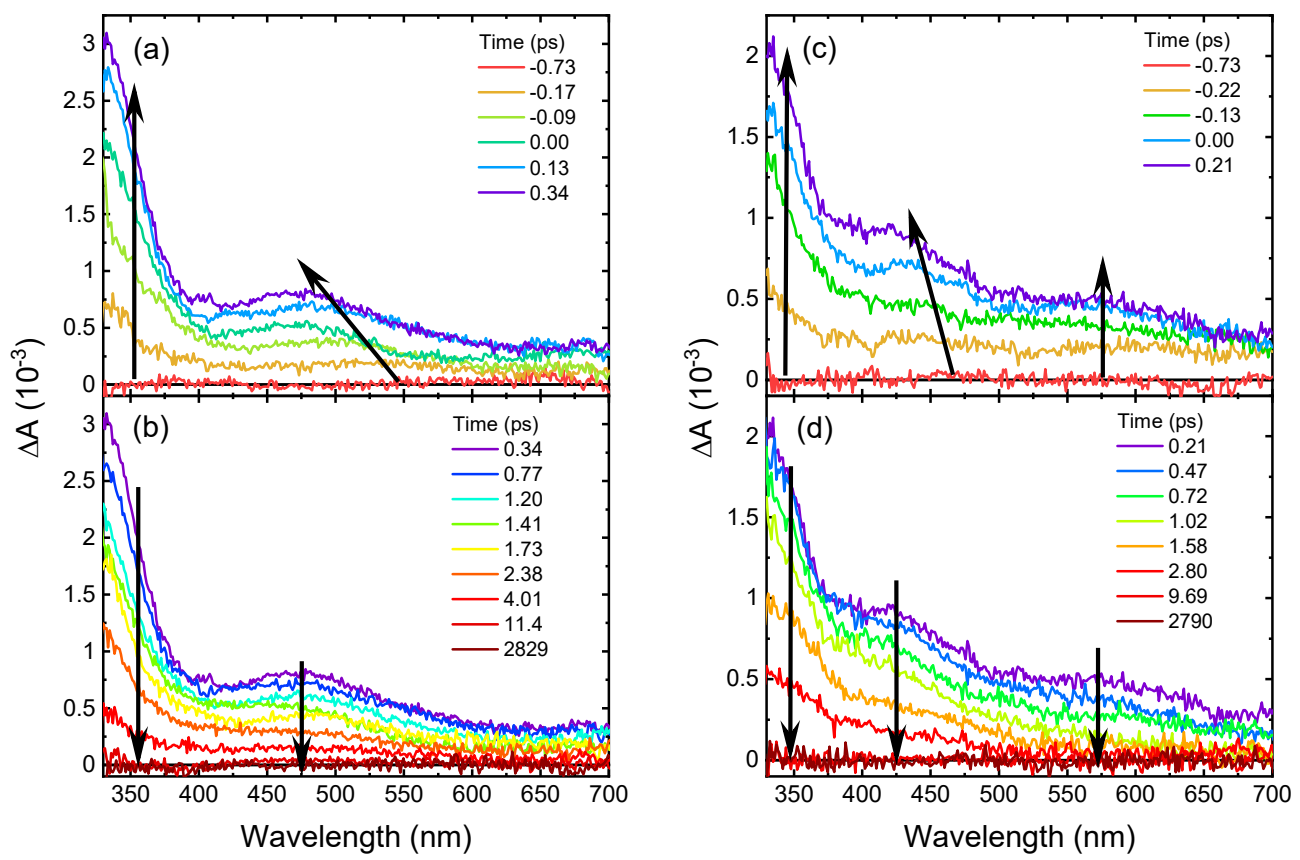

Figure 2. Spectral evolution of the transient absorption spectra of 7 dza (a-b) and GMP (c-d) following excitation at $267 \mathrm{~nm}$ in phosphate buffer $\mathrm{pH}$ 6.8. The arrows used in this figure are not meant to highlight specific probe wavelengths but the trend of the transient absorption bands.

Two- and three component sequential kinetic models were used to globally fit the transient absorption data for both 7dza and GMP. The two-component model yielded lifetimes of $0.2 \pm 0.1$ ps and $1.8 \pm 0.1 \mathrm{ps}$, and of $0.6 \pm 0.1 \mathrm{ps}$ and $1.4 \pm 0.2 \mathrm{ps}$, for $7 \mathrm{dza}$ and GMP respectively, while the three-component model yielded lifetimes of $0.3 \pm 0.1 \mathrm{ps}, 0.6 \pm 0.1 \mathrm{ps}$, and $1.9 \pm 0.1 \mathrm{ps}$ for $7 \mathrm{dza}$, and $0.6 \pm 0.1 \mathrm{ps}, 0.7 \pm 0.1 \mathrm{ps}$, and $2.7 \pm 0.1 \mathrm{ps}$ for GMP. Figure 3 shows representative kinetic decay traces over the initial 20 ps time window. The solid lines represent the best fit obtained through global and target analysis of the full broadband transient absorption data for each molecule. The evolution associated difference spectra (EADS) extracted from the global and targeted analyses of the broadband data are shown in Figure 4. 

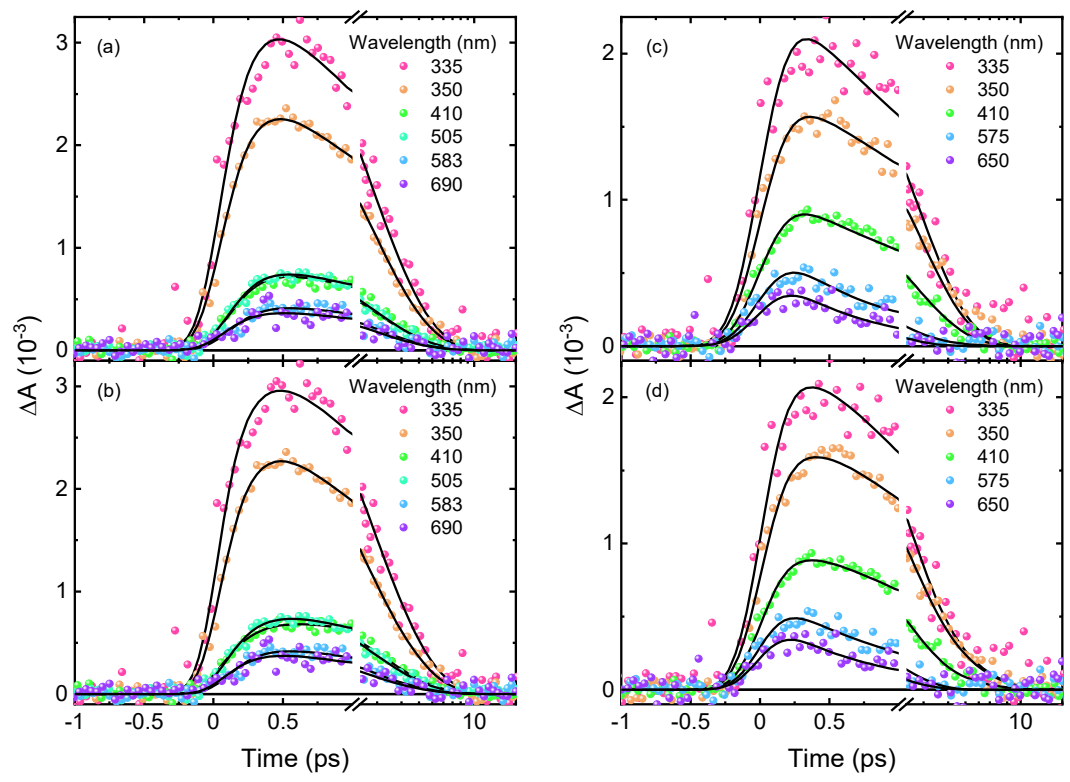

Figure 3. Representative kinetic decay traces of 7dza (a-b) and GMP (c-d) at select probe wavelengths globally fit with a two- $(\mathrm{a}, \mathrm{c})$ or a three-component $(\mathrm{b}, \mathrm{d})$ sequential kinetic model, respectively. Note, the abscissa is linear until $1 \mathrm{ps}$, after which a log scale is used.
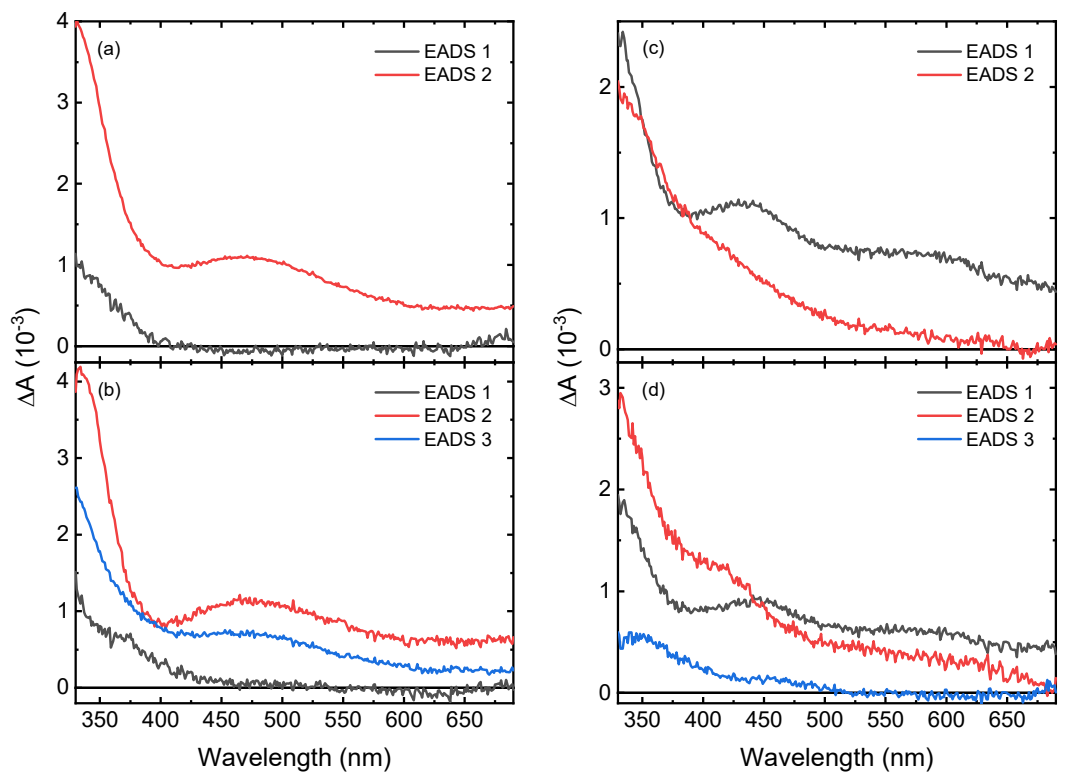

Figure 4. Evolution associated difference spectra of 7dza (a-b) and GMP (c-d) globally fit with a two- $(\mathrm{a}, \mathrm{c})$ or a three-component $(\mathrm{b}, \mathrm{d})$ sequential kinetic models, respectively. 

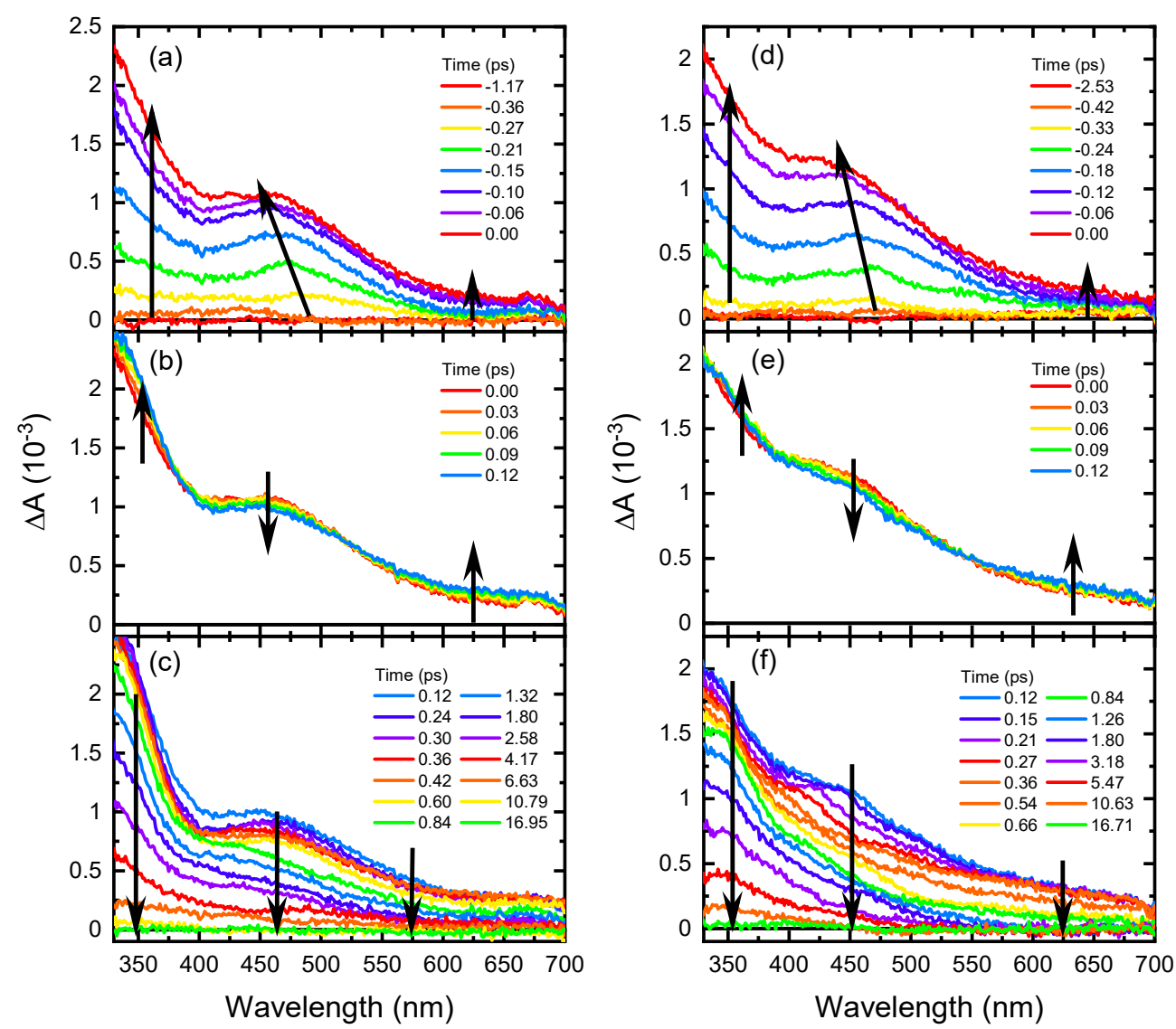

Figure 5. Spectral evolution of the transient absorption spectra of $7 d z a$ (a-c) and GMP (d-f) following excitation at $267 \mathrm{~nm}$ in $91 \%$ methanol and 9\% water. The arrows used in this figure are not meant to highlight specific probe wavelengths but the trend of the transient absorption bands.

The transient absorption spectra of 7dza and GMP were also collected in a mixture of $91 \%$ methanol and 9\% water (Figure 5). Initially, for $7 \mathrm{dza}$, an absorption band with maximum around $480 \mathrm{~nm}$ blue shifts to $460 \mathrm{~nm}$ within the cross correlation of the pump and probe beams, with a simultaneous growth of an absorption band with maximum below $330 \mathrm{~nm}$ (Figure 5a). Then, the band around $460 \mathrm{~nm}$ sightly decays within $0.1 \mathrm{ps}$, while the band below $330 \mathrm{~nm}$ and the tail at probe wavelengths above $525 \mathrm{~nm}$ simultaneously increase (Figure 5b). Apparent isosbestic points are observed at 380 and $520 \mathrm{~nm}$. From ca. 0.1 to $16 \mathrm{ps,} \mathrm{the} \mathrm{band} \mathrm{with} \mathrm{absorption} \mathrm{maximum} \mathrm{below}$ $330 \mathrm{~nm}$ starts to decay, while the band around $460 \mathrm{~nm}$ continues to decay. Both bands appear to decay with different rates (Figure 5c). No transient absorption signal is observed after a time delay 
of ca. 16 ps. For GMP, absorption bands with maxima below 330 and around 470 and $625 \mathrm{~nm}$ are initially observed within the cross correlation of the pump and probe beams (Figure $5 \mathrm{~d}$ ), with the band at $470 \mathrm{~nm}$ blue shifting to ca. $450 \mathrm{~nm}$. Then, the band around $450 \mathrm{~nm}$ sightly decays within $0.1 \mathrm{ps}$, while the band below $330 \mathrm{~nm}$ and the tail at probe wavelengths above $550 \mathrm{~nm}$ simultaneously increase (Figure 5e). Apparent isosbestic points are observed at 380 and $525 \mathrm{~nm}$. After that, the band with absorption maximum below $330 \mathrm{~nm}$ starts to decay, while the band around $450 \mathrm{~nm}$ continues to decay. Both bands appear to decay with different rates (Figure 5f). No transient absorption signal is observed after a time delay of ca. $16 \mathrm{ps.}$
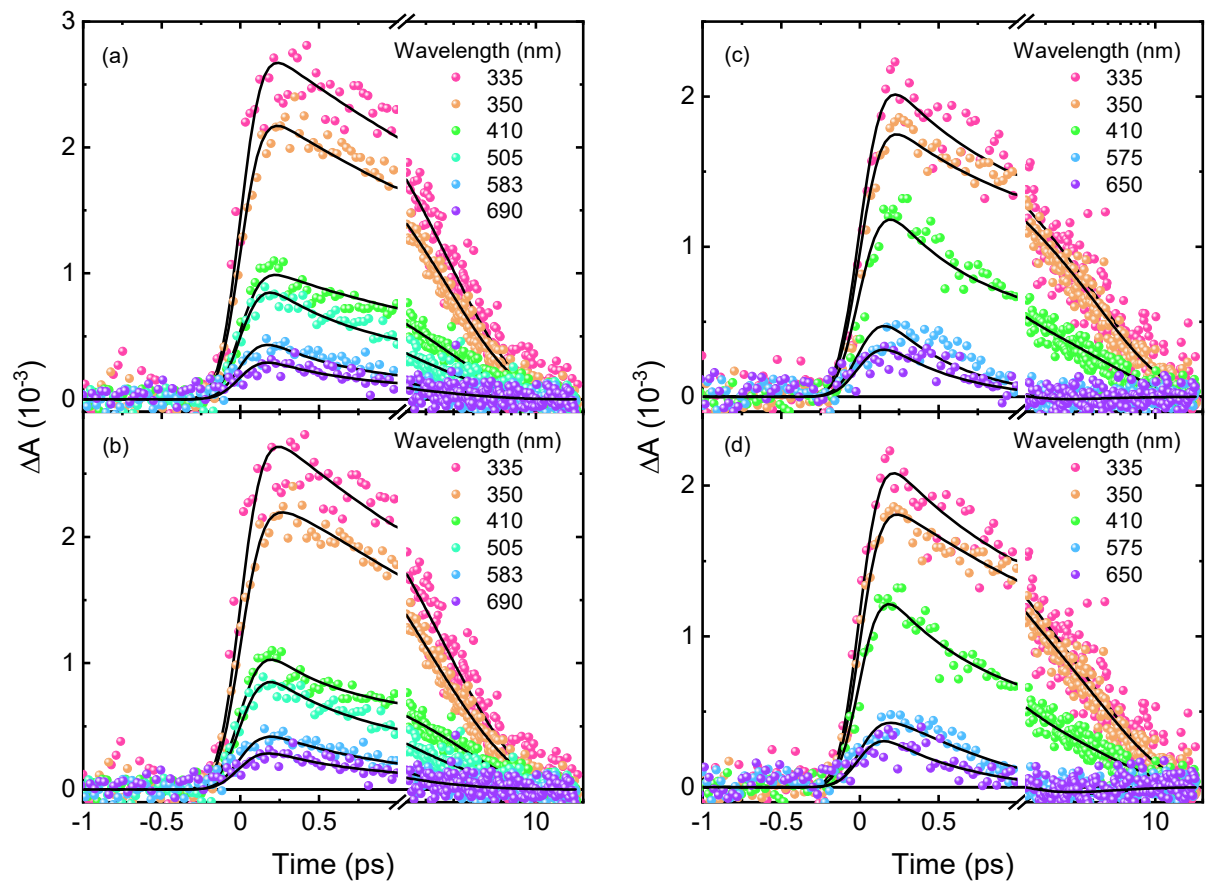

Figure 6. Representative kinetic decay traces of 7dza (a-b) and GMP (c-d) at select probe wavelengths in $91 \%$ methanol and $9 \%$ water globally fit with a two- $(a, c)$ or a three-component $(b, d)$ sequential kinetic model, respectively. Note, the abscissa is linear until 1 ps, after which a $\log$ scale is used.

Two- and three component sequential kinetic models were used to globally fit the transient absorption data for both 7dza and GMP in the mixture of 91\% methanol and 9\% water. The two- 
component model yielded lifetimes of $0.6 \pm 0.1 \mathrm{ps}$ and $2.8 \pm 0.2 \mathrm{ps}$, and of $0.5 \pm 0.1 \mathrm{ps}$ and $4 \pm 1$ ps, for 7dza and GMP respectively, while the three-component model yielded lifetimes of $0.3 \pm$ $0.1 \mathrm{ps}, 0.9 \pm 0.2 \mathrm{ps}$, and $3.1 \pm 0.2 \mathrm{ps}$ for $7 \mathrm{dza}$, and $0.3 \pm 0.1 \mathrm{ps}, 0.7 \pm 0.1 \mathrm{ps}$, and $4 \pm 1 \mathrm{ps}$ for GMP. Figure 6 shows representative kinetic decay traces over the initial $20 \mathrm{ps}$ time window. The solid lines represent the best fit obtained through global and target analysis of the full broadband transient absorption data for each molecule. The EADS extracted from the global and targeted analyses of the broadband data are shown in Figure 7.
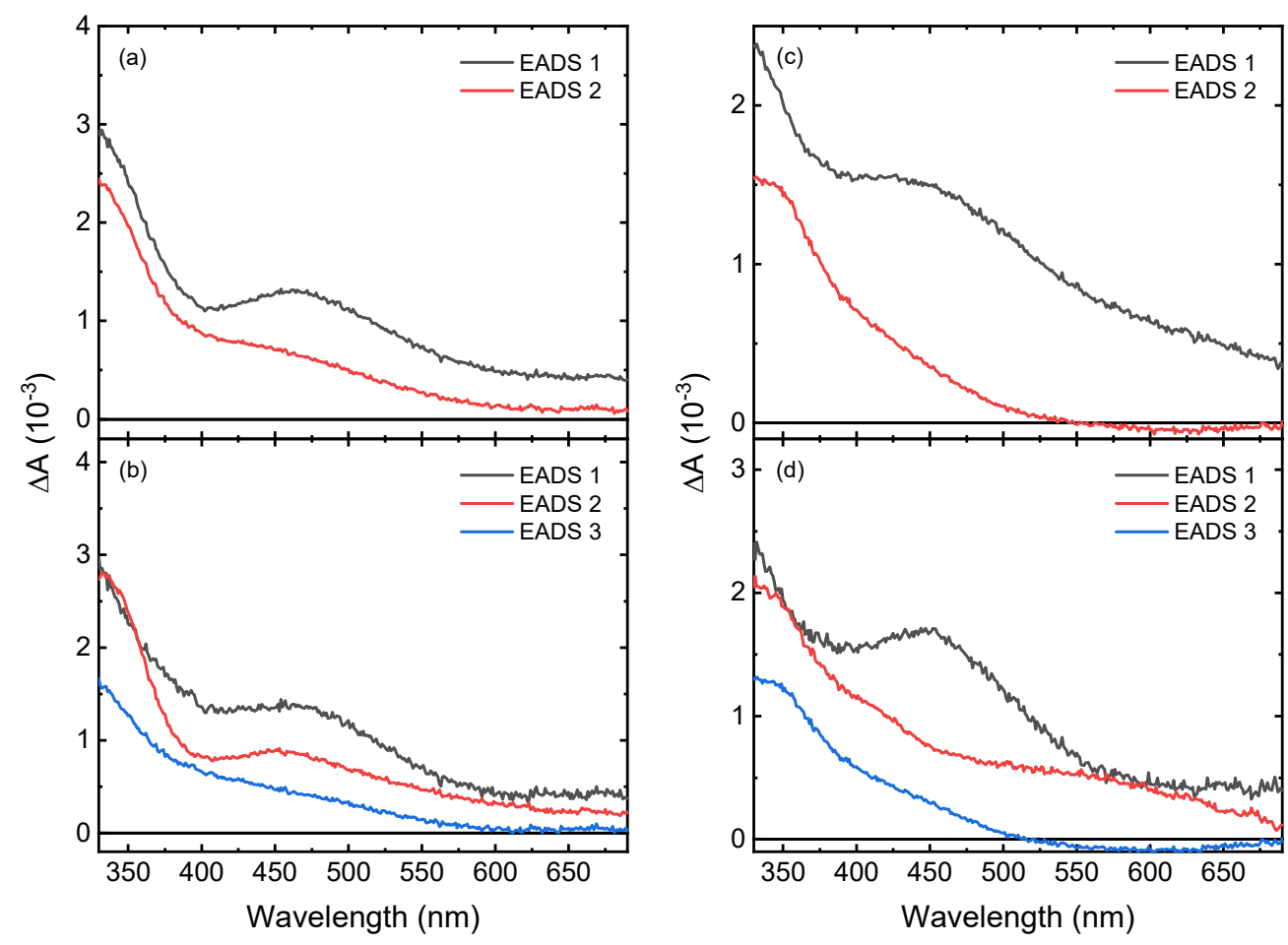

Figure 7. Evolution associated difference spectra of 7dza (a-b) and GMP (c-d) in a mixture of $91 \%$ methanol and $9 \%$ water globally fit with a two- $(\mathrm{a}, \mathrm{c})$ or a three-component $(\mathrm{b}, \mathrm{d})$ sequential kinetic models, respectively. 


\section{Discussion}

\section{A. Excited-state relaxation mechanism for $7 \mathrm{dza}$ in aqueous solution}

The absorptivity spectrum and the vertical excitation energies and oscillator strengths reported in Figure 1 and Table 1, respectively, suggest that both the $\mathrm{S}_{1}\left(\pi \pi^{*}, \mathrm{~L}_{\mathrm{a}}\right)$ and $\mathrm{S}_{2}\left(\pi \pi^{*}, \mathrm{~L}_{\mathrm{b}}\right)$ states are populated simultaneously following excitation of $7 \mathrm{dza}$ at $267 \mathrm{~nm}$ in aqueous solution. Note that direct population of the $\mathrm{S}_{3}\left(\pi \sigma^{*}\right.$, ICT $)$ cannot completely be ruled out within the accuracy of the calculations. However, less than ca. $0.6 \%$ of the initial excited state population is expected to reach this state directly given its small oscillator strength compared to those of the $\mathrm{S}_{1}\left(\pi \pi^{*}, \mathrm{~L}_{\mathrm{a}}\right)$ and $\mathrm{S}_{2}\left(\pi \pi^{*}, \mathrm{~L}_{b}\right)$ states. Hence, using a linear combination of two Gaussian functions to model the absorptivity spectrum of 7dza in the spectral region from ca. 230 to $330 \mathrm{~nm}$ (Figure S8a), it is estimated that about $95 \%$ of the initial excited state population reaches the $\mathrm{L}_{b}$ state, whereas only about $5 \%$ reaches the $\mathrm{L}_{\mathrm{a}}$ state. As shown in Figure $2 \mathrm{a}, \mathrm{b}$, the excited state population fully decays within about 12 ps and the broadband data can be satisfactorily fit with a two-component sequential kinetic model, yielding lifetimes of 0.2 and 1.8 ps. Thus, within a two-component model, we associate the first lifetime to two processes: internal conversion from the $\mathrm{Lb}_{\mathrm{b}}$ state to the $\mathrm{L}_{\mathrm{a}}$ state and simultaneous direct population of the $\mathrm{L}_{\mathrm{a}}$ state, both occurring within the cross-correlation of the pump and probe beams. This assignment may explain the blue shift in the transient absorption spectra observed in Figure 2a. As a result, the black EADS reported in Figure 4a would be primarily associated with the excited-state absorption of the $\mathrm{L}_{b}$ state. The second lifetime would then be assigned to internal conversion from the $\mathrm{L}_{\mathrm{a}}$ state to the ground state. The red EADS in Figure $4 \mathrm{a}$ would be associated with the excited-state absorption of the $\mathrm{La}$ state, from which population decays to the ground state in $1.8 \mathrm{ps}$. 
Using the three-component sequential kinetic model, the first lifetime of $0.3 \mathrm{ps}$ would be associated with internal conversion from the $\mathrm{L}_{b}$ state to the $\mathrm{L}_{a}$ state and simultaneous direct population of the $\mathrm{L}_{\mathrm{a}}$ state, and the black EADS reported in Figure $4 \mathrm{~b}$ would be associated with depopulation of $\mathrm{L}_{b}$ state with an apparent stimulated emission signal below $375 \mathrm{~nm}$. The red EADS in Figure $4 \mathrm{~b}$ would then be associated with the excited-state absorption of the $\mathrm{L}_{\mathrm{a}}$ state, while the blue EADS in Figure $4 \mathrm{~b}$ would be associated with the population of the $\mathrm{S}_{3}\left(\pi \sigma^{*}, \mathrm{ICT}\right)$ state. Results in a mixture of $91 \%$ methanol and $9 \%$ water reported in Section C provide further support of the involvement of the $\mathrm{S}_{3}\left(\pi \sigma^{*}, \mathrm{ICT}\right)$ state in the excited state relaxation mechanism of $7 \mathrm{dza}$. Currently, however, it is unclear whether one or both the $\mathrm{Lb}$ and $\mathrm{La}_{a}$ states can internally convert to the $\mathrm{S}_{3}\left(\pi \sigma^{*}\right.$, ICT) state outside the Franck-Condon region, or if the $\mathrm{S}_{3}\left(\pi \sigma^{*}\right.$, ICT) state is directly populated upon excitation at $267 \mathrm{~nm}$. Further quantum-chemical calculations are necessary to fully delineate the electronic relaxation mechanism for $7 \mathrm{dza}$.

\section{B. Excited-state relaxation mechanism for GMP in aqueous solution}

As observed for $7 \mathrm{dza}$, the absorptivity spectrum and the vertical excitation energies presented in Figure 1 and Table 1, respectively, suggest that both the $\mathrm{S}_{1}\left(\pi \pi^{*}, \mathrm{~L}_{\mathrm{a}}\right)$ and $\mathrm{S}_{2}\left(\pi \pi^{*}, \mathrm{~L}_{\mathrm{b}}\right)$ states are populated simultaneously upon excitation at $267 \mathrm{~nm}$ in aqueous solution, while direct population of the $S_{3}\left(\pi \sigma^{*}, I C T\right)$ state cannot be completely ruled out within the accuracy of the calculations. Note, however, that if the $S_{3}\left(\pi \sigma^{*}\right.$, ICT) state is directly populated upon $267 \mathrm{~nm}$ excitation, only $0.4 \%$ of the initial excited state population would be expected to reach this state given its small oscillator strength. Hence, the absorptivity spectrum of GMP from ca. 230 to 330 $\mathrm{nm}$ was modeled using a linear combination of two Gaussian functions (Figure S8b), as was done for $7 \mathrm{dza}$, to estimate the fraction of the initial excited state population that reaches the $\mathrm{S}_{1}\left(\pi \pi^{*}, \mathrm{~L}_{\mathrm{a}}\right)$ and $\mathrm{S}_{2}\left(\pi \pi^{*}, \mathrm{~L}_{b}\right)$ states. According to this analysis, about $79 \%$ of the initial population reaches the 
$\mathrm{L}_{b}$, whereas about $21 \%$ reaches the $\mathrm{L}_{\mathrm{a}}$ state. Then, assuming a two-component sequential model, the transient absorption data and the vertical excitation energies suggest that the first lifetime is most likely assigned to internal conversion from the $S_{1}\left(\pi \pi^{*}, L_{a}\right)$ state to the ground state. The population reaching the $\mathrm{L}_{b}$ state internally converts to the $\mathrm{L}_{a}$ state within the time resolution of our setup and cannot be clearly resolved. In other words, excitation of GMP at $267 \mathrm{~nm}$ populates both the $\mathrm{L}_{b}$ and $\mathrm{L}_{a}$ states simultaneously, however, the population reaching the $\mathrm{L}_{b}$ state internally converts to the $\mathrm{L}_{a}$ state in an ultrafast time scale. The population reaching the $\mathrm{L}_{\mathrm{a}}$ state then decays to the ground state with a $0.6 \mathrm{ps}$ lifetime. The decay of the excited state population in the $\mathrm{L}_{\mathrm{a}}$ state is followed by a slower decay of a broad transient absorption species with a lifetime of $1.4 \mathrm{ps}$. In principle, the second lifetime could be assigned to energy transfer from the vibrationally-excited ground state to the solvent molecules (i.e., vibrational cooling dynamics) or to a population decay from the $\mathrm{S}_{3}\left(\pi \sigma^{*}, \mathrm{ICT}\right)$ state to the ground state. Then, within a two-component model, the black EADS in Figure $4 c$ would primarily represent the excited-state absorption spectrum of the $\mathrm{L}_{\mathrm{a}}$ state, whereas the red EADS would primarily represent the absorption spectrum of the vibrationallyexcited ground state or that of the $\mathrm{S}_{3}\left(\pi \sigma^{*}, \mathrm{ICT}\right)$ state.

Assuming a three-component kinetic model, the black EADS associated to the first lifetime of $0.6 \mathrm{ps}$ would be assigned to the simultaneous population of both the $\mathrm{L}_{b}$ and $\mathrm{L}_{\mathrm{a}}$ states (Figure 4d), while the red EADS associate to the second lifetime of $0.7 \mathrm{fs}$ would be assigned to the excited absorption spectrum of the relax La state (Figure 4d). Here again, the blue EADS associated with the longer lived transient species with a lifetime of 2.7 ps would be assigned to the absorption spectrum of the vibrationally-excited ground state or that of the $S_{3}\left(\pi \sigma^{*}\right.$, ICT) state (Figure $4 d$ ). Results in a 91\% methanol and 9\% water mixture reported in Section C support the involvement of the $\mathrm{S}_{3}\left(\pi \sigma^{*}, \mathrm{ICT}\right)$ state in the excited state relaxation mechanism of GMP. As for $7 \mathrm{dza}$, it is 
currently unclear whether one or both the $\mathrm{L}_{b}$ and $\mathrm{L}_{\mathrm{a}}$ states can internally convert to the $\mathrm{S}_{3}\left(\pi \sigma^{*}\right.$, ICT) state outside the Franck-Condon region, or if the $S_{3}\left(\pi \sigma^{*}, I C T\right)$ state is directly populated upon excitation at $267 \mathrm{~nm}$. Quantum chemical calculations should be able to provide insights about this puzzle.

\section{Excited state dynamics of 7dza and GMP in a mixture of $91 \%$ methanol and $9 \%$ water}

In an effort to clarify whether the longer lived lifetime in both 7dza and GMP is associated to vibrational cooling in the ground state or to the decay of the $\mathrm{S}_{3}\left(\pi \sigma^{*}, \mathrm{ICT}\right)$ state, both molecules were dissolved in a mixture of $91 \%$ methanol and $9 \%$ water and their transient absorption spectra were collected. Methanol was selected as a solvent because earlier experiments by Cheng et al. ${ }^{25}$ with 2'-deoxyguanosine have suggested an increase in excited state population of a weakly emissive species fluorescing around $500 \mathrm{~nm}$ and decaying within a few picoseconds, which the authors tentatively assigned to an excited singlet state with $\pi \sigma^{*}$ or ICT character. Importantly, this state was observed in both femtosecond fluorescence up-conversion and transient absorption experiments for both 2'-deoxyguanosine (dGuo) and 2'-deoxyguanosine 5'-monophosphate (dGMP) in PBS and in methanol solutions. Unfortunately, GMP and 7dza are not soluble enough for study in pure methanol but are sufficiently soluble in a mixture of $91 \%$ methanol and $9 \%$ water. Excitation of 7dza and GMP in this mixture results in the transient absorption spectra shown in Figure 5. As in aqueous solution, we assume that both the $\mathrm{Lb}_{\mathrm{b}}$ and $\mathrm{L}_{\mathrm{a}}$ states of $7 \mathrm{dza}$ and GMP are directly populated at $267 \mathrm{~nm}$ because the steady-state absorption spectra are practically identical in both solutions (Figure $\mathrm{S} 1)$. Here again, we cannot rule out or evidence whether the $\mathrm{S}_{3}\left(\pi \sigma^{*}, \mathrm{ICT}\right)$ state is populated directly upon excitation at $267 \mathrm{~nm}$ in the $91 \%$ methanol and $9 \%$ water mixture. 
For 7dza, the magnitude of both lifetimes increases in going from aqueous solution to the methanol/water mixture when using a two-component sequential model. Within this model, the black EADS reported in Figure 7a would be associated with the excited state absorption spectrum of the $\mathrm{L}_{\mathrm{a}}$ state, assuming that internal conversion from the $\mathrm{L}_{b}$ to $\mathrm{L}_{a}$ state occurs in a shorter time scale than the time resolution of our transient absorption spectrometer. The second lifetime and the EADS associated to it (red spectrum in Figure 7a) would be assigned to the absorption spectrum of the $\mathrm{S}_{3}\left(\pi \sigma^{*}\right.$, ICT) state, which has an absorption maximum below $330 \mathrm{~nm}$ and an absorption tail that extends all the way to ca. $600 \mathrm{~nm}$. Using a three-component sequential model, the first lifetime, and the black EADS in Figure 7b, would be associated with the initial population of the $\mathrm{L}_{b}$ and $\mathrm{L}_{\mathrm{a}}$ states within the cross correlation of the pump and probe beams. The second lifetime, and the red EADS spectrum shown in Figure 7b, would be associated with the decay of the $\mathrm{L}_{a}$ minimum, while the third lifetime, and the blue EADS in Figure 7b, would be associated with the decay of the $\mathrm{S}_{3}\left(\pi \sigma^{*}, \mathrm{ICT}\right)$ state to the ground state. As in aqueous solution, it is currently unclear whether one or both the $\mathrm{Lb}_{b}$ and $\mathrm{L}_{a}$ states can internally convert to the $\mathrm{S}_{3}\left(\pi \sigma^{*}\right.$, ICT) state outside the FranckCondon region, or if the $\mathrm{S}_{3}\left(\pi \sigma^{*}, \mathrm{ICT}\right)$ state is directly populated upon excitation at $267 \mathrm{~nm}$.

The transient absorption spectra of GMP in a mixture of $91 \%$ methanol and $9 \%$ water is shown in Figure 5d,e,f. Using a two-component sequential model, the first lifetime is practically unchanged compared to aqueous solution, while the second lifetime significantly increases. As for 7dza, the first lifetime would be associated with the simultaneous population of the $\mathrm{Lb}_{b}$ and $\mathrm{La}_{\mathrm{a}}$ states, while the corresponding black EADS in Figure 7c would be associated to the excited state absorption of the $\mathrm{La}_{\mathrm{a}}$ state due to sub-100 fs internal conversion from the $\mathrm{Lb}_{\mathrm{b}}$ to the $\mathrm{La}_{\mathrm{a}}$ state. The second lifetime would be associated with the decay of the $\mathrm{S}_{3}\left(\pi \sigma^{*}\right.$, ICT) state to the ground state, while the corresponding red EADS in Figure 7c would be associated with the absorption spectrum 
of the vibrationally-excited ground state or that of the $\mathrm{S}_{3}\left(\pi \sigma^{*}\right.$, ICT) state. We remark at this point that the red EADS exhibits an absorption maximum around $330 \mathrm{~nm}$, with an absorption tail extending to ca. $550 \mathrm{~nm}$.

Assuming a three-component sequential model, the first lifetime of $0.3 \mathrm{ps}$ would be assigned to the simultaneous population of both the Lb and La states (black EADS in Figure 7d), while the second lifetime of 0.7 ps would be assigned to the excited absorption spectrum of the $\mathrm{La}_{\mathrm{a}}$ minimum or internal conversion of the $\mathrm{L}_{\mathrm{a}}$ state to the $\mathrm{S}_{3}\left(\pi \sigma^{*}, \mathrm{ICT}\right)$ state (red EADS in Figure $\left.7 \mathrm{~d}\right)$. The third lifetime of 4 ps would be associated with the absorption spectrum of the vibrationallyexcited ground state or that of the $\mathrm{S}_{3}\left(\pi \sigma^{*}, \mathrm{ICT}\right)$ state (blue EADS in Figure $7 \mathrm{~d}$ ).

For both 7dza and GMP, irrespective of the solvent used, we favor the assignment of the longer picosecond lifetime to the decay of the $\mathrm{S}_{3}\left(\pi \sigma^{*}\right.$, ICT) state to the ground state, instead of vibrational cooling dynamics in the ground state, based on the following observations. Firstly, vibrational cooling is often probe wavelength dependent, ${ }^{35}$ with the observation of longer lifetimes at shorter probe wavelengths. We have normalized the probe wavelengths from 330 to $360 \mathrm{~nm}$ for both $7 \mathrm{dza}$ and GMP in both aqueous solution and the $91 \%$ methanol and $9 \%$ water mixture (Figure S9) and observed that all probe wavelengths exhibit the same decay lifetime within the experimental uncertainties. Secondly, the EADS associated with the longer picosecond lifetime has a maximum near $330 \mathrm{~nm}$ and an absorption tail that extend all the way to visible probe wavelengths. It is very unlikely that ultrafast internal conversion from a singlet excited state in both molecules will populate a vibrationally-excited ground state that would absorb all the way to the visible. Thirdly, as observed by Cheng et al. ${ }^{25}$ for dGuo in methanol, the longer picosecond lifetime significantly increases in going from aqueous solution to a methanol/water mixture, which was explained by these authors as due to the more selective and stronger hydrogen bond accepting 
capacity of methanol than the water solvent. This increased hydrogen bond accepting capacity may allow a more effective structural and energy relaxation of the $S_{3}\left(\pi \sigma^{*}, I C T\right)$ minimum, hence, increasing the relative energy barrier to access a conical intersection with the ground state. We remark that such a conical intersection has not been identified for 7dza or GMP thus far but has been characterized for an analogous state in both adenosine ${ }^{33}$ and 8-oxo-deoxyguanosine, ${ }^{34}$ recently. Tuna and Domcke have termed this relaxation process as an electron-driven protontransfer pathway. ${ }^{33,34}$ It should be remarked that we are not ruling out the likely possibility that vibrational cooling dynamics occur in the ground state upon ultrafast internal conversion from the $\mathrm{L}_{\mathrm{a}}$ excited singlet state. However, the transient absorption results presented in this study do not support that vibrational cooling is a primary relaxation pathway observed in the spectral probe region from 330 to $700 \mathrm{~nm}$ for either $7 \mathrm{dza}$ or GMP in aqueous solution or in a methanol-water mixture.

\section{Comparison of the excited state dynamics of GMP with earlier time resolved measurements}

The broadband transient absorption results reported in this study for GMP generally agree well with earlier work done by Karunakaran and Cheng, ${ }^{24,25}$ who observed similar transient absorption spectra for GMP as those reported in Figure 2c,d. However, there is a slight discrepancy in the relative intensity of the UV and visible transient absorption bands around 425 and $600 \mathrm{~nm}$, respectively. Our results show higher intensity for the absorption bands at smaller probe wavelengths than ca. $500 \mathrm{~nm}$, whereas work by Karunakaran and Cheng show approximately equal intensity for both bands at ca. 450 and $600 \mathrm{~nm}$. This may be associated to the different instrument response function (IRF) of the transient absorption setups, particularly if the IRF of both of their systems is shorter than that of our setup of ca. $250 \pm 50 \mathrm{fs}$. In such a case, the amplitude of the 
visible absorption band assigned to excited-state absorption of the $\mathrm{L}_{\mathrm{a}}$ state may be underestimated in our transient absorption experiments because a significant fraction of this excited-state population would have decayed within the cross-correlation of the pump and probe beams. Another factor that could explain the discrepancy between the relative intensities of the 450 and $600 \mathrm{~nm}$ absorption bands for GMP in Figure 2c,d versus in experiments by Karunakaran et al. and Cheng et al. could be due to an over subtraction of the solvent signal at probe wavelengths smaller than ca. $500 \mathrm{~nm}$. Solvent signal subtraction is required when a relatively high photon density is used for excitation at $267 \mathrm{~nm}$, resulting in the formation of hydrated electrons as a consequence of two-photon ionization of the water solvent. ${ }^{36}$ It should be remarked at this point that we have also observed a signal from the water solvent in the spectral probe region from 320 to $450 \mathrm{~nm}$ that increases linearly with an increase in the pump photon density at $267 \mathrm{~nm}$ (see Methods section below). In our experience, the combination of the linear and quadratic-dependent signals originating from the solvent makes the correction of the transient absorption spectra of GMP (or any other molecule excited at $267 \mathrm{~nm}$ in aqueous solution) for these solvent signals a challenge because at probe wavelengths around $450 \mathrm{~nm}$ both linear and quadratic-dependent solvent signals overlap. This is the reason for which we dedicated considerable effort at optimizing the experimental conditions to eliminate the need to correct the transient absorption data of GMP and $7 \mathrm{dza}$ for the linear and quadratic-dependent solvent signals.

We note that recent computational studies for guanine monomers at the CASSCF and DFT levels of theory have suggested that upon moving away from the FC region, the $\mathrm{L}_{\mathrm{b}}$ state quickly reaches a conical intersection with the lower lying $\mathrm{La}_{\mathrm{a}}$ state. ${ }^{24,28,29}$ This relaxation pathway is thought to occur in less than $50 \mathrm{fs}$. Hence, it is likely that such ultrafast internal conversion process cannot be resolved with our experimental setup, as we proposed above. We note that previous 
experimental investigations using fs-transient absorption and fs-fluorescence up-conversion spectroscopy have reported three lifetimes for GMP, ${ }^{24-26}$ which is the reason for why we also modeled the transient absorption data using a three-component kinetic model in addition to using a two-component model. Obviously, as shown in Figures 3 and 6, our broadband data can be equally well fitted with three lifetimes of similar magnitude as those used in the previous works. In fact, the transient absorption data for GMP fits equally well using the lifetimes reported by either Karunakaran or Cheng et al. (see Figure S10). ${ }^{24,25}$ The observation that either a two- or a three-component kinetic model, or even a multiple set of three lifetimes (i.e., ours or those reported by Karunakaran and Cheng), can fit well the broadband transient absorption data for GMP highlights the fact that the transient absorption species overlap strongly in the spectral region from 330 to $700 \mathrm{~nm}$ and that all the processes occur within a similar time scale. It also highlights the difficulty of extracting accurate lifetimes from a global and target analysis of the broadband data when the magnitude of the lifetime for each process is close to one another. Therefore, the exact magnitude of the lifetimes reported in this study (or in other broadband investigations) should be taken with some caution (i.e., taking these difficulties in consideration) because they depend on the extend of spectral overlap of the transient absorption species, particularly if they evolve within a similar time scale, and on the signal/noise ratio of the collected data.

In their broadband transient absorption experiments, Karunakaran et al. reported an ultrafast lifetime of about $200 \mathrm{fs}$, which was assigned to movement of the wavepacket along the $\mathrm{L}_{\mathrm{a}}$ state potential energy surface from a planar region to a shallow minimum. ${ }^{24}$ From this minimum, the excited population reaches a conical intersection with the ground state, through ring puckering at the $\mathrm{C} 2$ atom, ${ }^{24}$ in agreement with recent calculations for GMP. ${ }^{28}$ This deformation of planarity allows for internal conversion back to the ground state, corresponding with the second lifetime of 
approximately $800 \mathrm{fs}$. Not surprisingly, the average of both lifetimes agrees well with our reported lifetime of 0.6 ps using a two-component model, suggesting this lifetime is simply the weighted mean of the two exponential lifetimes reported by Karunakaran et al. Hence, the 0.6 ps lifetime reported in our study can be directly associated with the $\tau_{1}$ and $\tau_{2}$ dynamics reported in this early work (i.e., wavepacket motion along the almost flat $\mathrm{L}_{a}$ potential energy surface $\left(\tau_{1}\right)$ and internal conversion through the $\mathrm{La}_{\mathrm{a}} / \mathrm{S}_{0}$ conical intersection $\left(\tau_{2}\right)$ ). This is further supported by the blueshift in the transient absorption band of GMP within this time delay (Figures $2 \mathrm{c}$ and $5 \mathrm{c}$ ). The third lifetime of ca. 2 ps, was assigned to vibrational cooling in the ground state, ${ }^{24}$ while Cheng et al. ${ }^{25}$ tentatively assigned it to an excited singlet state with $\pi \sigma^{*}$ or ICT character. As discussed above, we assign the third lifetime to the decay of the $\mathrm{S}_{3}\left(\pi \sigma^{*}\right.$, ICT) state to the ground state.

\section{E. Comparison of the excited-state dynamics in 7dza and GMP}

As shown in Scheme 1, substitution of the nitrogen atom at the seven position of the guanine chromophore by a $\mathrm{C}-\mathrm{H}$ group gives rise to the $7 \mathrm{dza}$ nucleobase. One of the motivations of this study is to probe if this simple substitution affects the excited state dynamics observed in the guanine monomers, particularly in GMP. Hence, we performed steady-state, time-resolved, and computational calculations for both molecules under equal experimental and computational conditions. Scheme 2 depicts a general relaxation mechanism $\left(\mathrm{L}_{b} \rightarrow \mathrm{L}_{a} \rightarrow{ }^{1} \pi \sigma^{*}(\mathrm{ICT}) \rightarrow \mathrm{S}_{0}\right)$ that we propose may describe the relaxation dynamics for both 7dza and GMP. We remark that it is currently unknown whether some of the population in the $\mathrm{L}_{b}$ and/or $\mathrm{L}_{\mathrm{a}}$ states can internally convert to the ${ }^{1} \pi \sigma^{*}(\mathrm{ICT})$ state, which is the reason that we drop the labeling of singlet states as $\mathrm{S}_{1}, \mathrm{~S}_{2}$, and $\mathrm{S}_{3}$, hereafter. It is also unknown whether a fraction of the population reaching the $\mathrm{L}_{\mathrm{a}}$ state can internally convert to the ground state, through the known conical intersection, ${ }^{24}$ in competition with a fractional population reaching the ${ }^{1} \pi \sigma^{*}$ (ICT) state. It is, however, clear that the ${ }^{1} \pi \sigma^{*}(\mathrm{ICT})$ 
state internally converts to the ground state in both molecules, suggesting that there is a conical intersection between the ${ }^{1} \pi \sigma^{*}$ (ICT) state and the ground state in both molecules, similar to what has been observed for an analogous state in adenosine ${ }^{33}$ and 8-oxo-deoxyguanosine. ${ }^{34}$ It is also currently unknown if the putative conical intersection between the ${ }^{1} \pi \sigma^{*}(\mathrm{ICT})$ state and the ground state is lower or higher in energy than the conical intersection between the $\mathrm{L}_{\mathrm{a}}$ and the ground state, and whether the $\mathrm{L}_{\mathrm{a}}$ minimum ( $\mathrm{La}$ min in Scheme 2 ) is lower or higher in energy than the ${ }^{1} \pi \sigma^{*}$ (ICT) minimum $\left({ }^{1} \pi \sigma^{*}(\mathrm{ICT})_{\min }\right.$ in Scheme 2$)$. These uncertainties in the order of the excited states and topology of the potential energy surfaces outside the Franck-Condon region are highlighted with a rectangle and question mark symbols in Scheme 2. Hence, at first sight, the general electronic relaxation mechanisms for both molecules appear to be qualitatively similar. However, the global and target analyses reported in this study suggest that there are quantitative differences between both molecules.

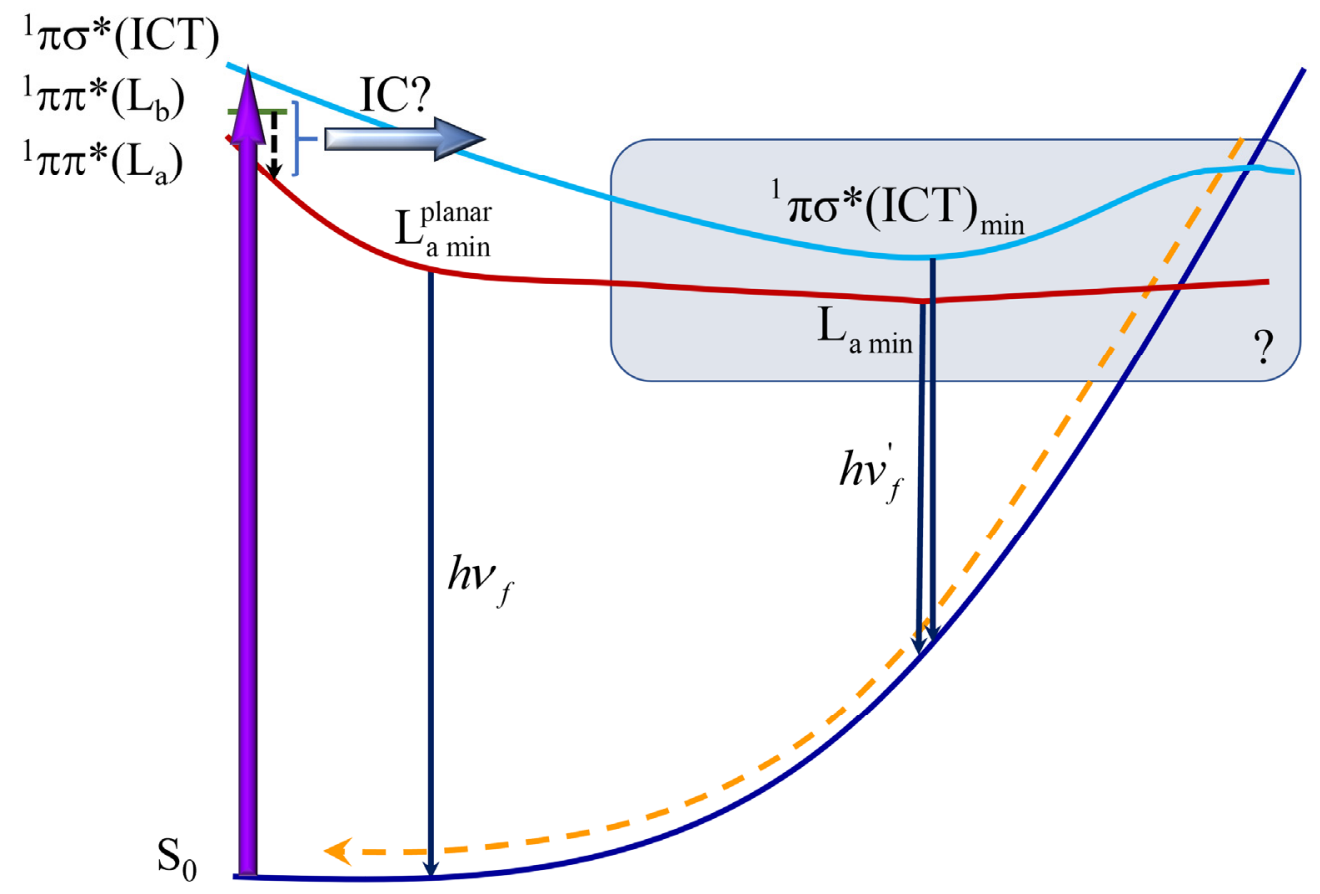

Scheme 2. Proposed Jabłoński diagram depicting the general electronic relaxation mechanism for 7dza and GMP. See discussion in Section E for details. 
For $7 \mathrm{dza}$, we propose that internal conversion from the $\mathrm{L}_{b}$ to $\mathrm{L}_{\mathrm{a}}$ state occurs within ca. 0.2 to $0.3 \mathrm{fs}$ in aqueous solution, whereas we propose that this internal conversion pathway cannot be resolved for GMP with our experimental setup (i.e., it should occur in a $<100 \mathrm{fs}$ time scale). This suggests that there may be a small energy barrier to access the conical intersection between the $\mathrm{L}_{b}$ and $\mathrm{La}_{\mathrm{a}}$ states in $7 \mathrm{dza}$ that is relatively larger than in GMP. According to our assignments, internal conversion from the $\mathrm{L}_{\mathrm{a}}$ to the ground state, or to the ${ }^{1} \pi \sigma^{*}$ (ICT) state, and the decay of the ${ }^{1} \pi \sigma^{*}(\mathrm{ICT})$ state occur within about the same lifetimes in both $7 \mathrm{dza}$ and GMP. This suggests that there are similar barriers to both access the conical intersection between the La state and the ground state (or ${ }^{1} \pi \sigma^{*}(\mathrm{ICT})$ state) and to access the conical intersection between the ${ }^{1} \pi \sigma^{*}(\mathrm{ICT})$ state and the ground state in both molecules. Comparisons of the excited state dynamics for both molecules at select probe traces are shown in Figures S11 and S12, supporting the idea that the overall relaxation dynamics are slower in 7dza than in GMP in aqueous solution and in a mixture of $91 \%$ methanol and $9 \%$ water.

\section{Conclusions}

In this study, steady-state and time-resolved absorption experiments were combined with vertical excitation energies to reveal the excited-state dynamics of $7 \mathrm{dza}$ in aqueous solution and in a methanol-water mixture upon excitation at $267 \mathrm{~nm}$. The results were compared directly with those obtained for GMP under equal experimental and computational conditions. It was shown that substitution of a single nitrogen for a methine $(\mathrm{C}-\mathrm{H})$ group at position seven of the guanine moiety stabilizes the ${ }^{1} \pi \pi^{*} \mathrm{~L}_{b}$ and $\mathrm{L}_{\mathrm{a}}$ states of $7 \mathrm{dza}$ relative to those of GMP, whereas the internal conversion of the ${ }^{1} \pi \sigma^{*}$ (ICT) state to the ground state occurs with a similar lifetime of a few picoseconds. Additionally, the kinetic analysis proposes that the internal conversion lifetime is longer because of this $\mathrm{N} 7$-for- $\mathrm{C} 7 \mathrm{H}$ substitution, which lends support to the idea that there is a 
change in the topology of the potential energy surfaces upon this simple substitution. Multiconfigurational excited-state calculations and molecular dynamics simulations are necessary to provide further insights about the electronic relaxation mechanism of both 7dza and GMP, particularly with regards to the participation of a ${ }^{1} \pi \sigma^{*}(\mathrm{ICT})$ state in the few picoseconds relaxation dynamics.

\section{Experimental and Computational Methodology}

\section{A. Materials and steady-state spectroscopy}

Guanosine 5'-monophosphate disodium salt (GMP, 97\% purity) and 7-deazaguanosine (7dza, 98\% purity) were purchased from Acros and Biosynth Carbosynth, respectively, and were used as received. We selected GMP instead of guanosine because it has better solubility in aqueous solution and the excited state dynamics of the guanine nucleosides and nucleotides have been demonstrated to be the same by Cheng et al. ${ }^{25}$ Phosphate buffer solutions with total phosphate concentration of $20 \mathrm{mM}$ from monosodium and disodium phosphate salts dissociated in ultrapure water (Millipore) were freshly prepared the day of each laser irradiation experiment. The $\mathrm{pH}$ of the solution was adjusted using $0.1 \mathrm{M}$ solutions of $\mathrm{NaOH}$ and $\mathrm{HCl}$ to the desired $\mathrm{pH}$ of $6.8( \pm 0.1$ $\mathrm{pH}$ units). Samples in the methanol mixture were prepared by first dissolving GMP and 7dza in ultrapure water to yield a concentrated stock solution. A small amount of each concentrated solution was added to methanol (Fisher Scientific, HPLC grade), resulting in a final mixture of 91\% methanol and 9\% water by volume for each sample. Steady-state absorption and emission were recorded using a Cary 100 and Cary Eclipse spectrometer, respectively. Emission spectra were obtained using a PMT voltage of $800 \mathrm{~V}$ with $5 \mathrm{~nm}$ slit widths and a scan rate of $20 \mathrm{~nm} / \mathrm{min}$. 


\section{B. Quantum chemical calculations}

Quantum chemical calculations were performed using Gaussian 16 suite of programs. ${ }^{37}$ Ground state optimizations were performed at the B3LYP/IEFPCM/6-311+G(d,p) level of theory for both 7dza and GMP in water. For each molecule, the calculations were performed with the sugar in both the syn- and anti-conformations (see Tables S1 and S2). A sodium atom was added as a counterion in the calculations of GMP to neutralize the negative charge of the phosphate group. Vertical excitation energies were calculated at the TD-PBE0/IEFPCM/6-311+G(d,p) level of theory. A polarizable continuum model (PCM) using the integral equation formalism (IEF) was included in the ground state optimization and for the calculations of the vertical excitation energies to model the bulk solvent dielectric effect of water on each molecule. ${ }^{38,39}$

\section{Transient absorption spectroscopy}

The experimental setup and data analysis used for the femtosecond broadband transient absorption spectroscopy (TAS) technique have been described in great deal elsewhere. ${ }^{40-42}$ Briefly, the TAS spectrometer (Helios, Ultrafast Systems) make use of a Ti:Sapphire oscillator (Vitesse, Coherent), which seeds a regenerative amplifier (Libra-HE, Coherent) producing 100 fs pulses, centered at $800 \mathrm{~nm}$, and with a $1 \mathrm{kHz}$ repetition rate. Generation of the $267 \mathrm{~nm}$ excitation pulse

was done as described previously, ${ }^{7}$ by pumping an optical rail kit (FKE series, EKSMA optics). A translating $2 \mathrm{~mm} \mathrm{CaF}_{2}$ window was used to generate the white light continuum in the spectral probe window from 320 to $700 \mathrm{~nm}$.

The absorbance of the sample solutions in aqueous solution and the methanol/water mixture at the excitation wavelength were matched at 1.3 for both GMP and 7dza during TAS experiments. The homogeneity of the solutions in a $2 \mathrm{~mm}$ path length fused silica cell was 
maintained by continuous stirring with a Teflon-coated magnetic stirbar. To minimize the putative contamination of the transient signals by the formation of any photoproducts absorbing at $267 \mathrm{~nm}$, freshly prepared samples were used, and frequently refreshed over the course of data acquisition such that the absorption of the samples at $267 \mathrm{~nm}$ did not change by more than 3 percent.

Data collection made use of a home-made LabView program, while global and target data analyses were performed using the Glotaran graphical user interface to the R-package TIMP software ${ }^{43}$ The full multidimensional data set for both GMP and 7dza was globally fit using a two or a three compartment sequential kinetic model convoluted with a Gaussian instrument response function of $250 \pm 50$ fs (FWHM). The evolution associated difference spectra (EADS) were extracted from the global and target analysis. ${ }^{43}$

\section{Experimental pump-probe conditions used to eliminate the two-photon hydrated electron signal from the buffer solvent}

In order to eliminate the need to correct for the two-photon ionization of water upon excitation of the sample solutions at $267 \mathrm{~nm},{ }^{36}$ the photon densities were adjusted to ensure no parasitic signal from the characteristic solvated electron ${ }^{44}$ were observed. To accomplish this, the probe beam size was kept constant at ca. 100 microns $(0.110 \pm 0.005 \mathrm{~mm})$, while the pump beam size was increased to ca. $1.2 \mathrm{~mm}(1.160 \pm 0.005 \mathrm{~mm})$ using the knife edge method to obtain the $1 / \mathrm{e}^{2}$ beam diameter. ${ }^{45}$ Briefly, a razor blade and micrometer translation stage is used to cover $15 \%$ and $85 \%$ of the beam power while noting the micrometer position, where the difference between the two measurements yields the desired beam diameter. The reported value is an average of a series of three measurements. Once the pump beam size was widened to the desired beam size, the average pump intensity was increased to $2 \mu \mathrm{J}$ at the cell to improve signal to noise of the recorded data. An average photon density or quantum flux of $2.5 \times 10^{17}$ photons $/ \mathrm{s} \cdot \mathrm{cm}^{2}$ was estimated and is 
further detailed in the SI. The fraction of photons absorbed by 7dza or GMP was maximized by using an absorbance of 1.3 at $267 \mathrm{~nm}$, while simultaneously eliminating the formation of solvated electron signal. This can be observed in Figure 8, where the neat buffer solvent and adenosine 5'monophosphate (AMP) were used to verify that no hydrated electron signal was observed under the experimental conditions used in this work.

We note that the broadband data for AMP can be globally fitted using a mono-exponential kinetic model (Figure 9; see also Figure S13), yielding a lifetime of $(0.39 \pm 0.02)$ ps. Interestingly, this value compares favorably with the lifetime reported by Kohler and co-workers in two independent studies for AMP in aqueous buffer solution of $(0.30 \pm 0.02) \mathrm{ps}^{36}$ and $(0.33 \pm 0.03)$ ps. ${ }^{46}$ However, in their broadband transient absorption experiments exciting at $267 \mathrm{~nm}$, Kwok et al. ${ }^{47}$ reported two lifetimes of 0.13 and $0.45 \mathrm{ps}$ for adenosine in aqueous solution, whereas Pecourt et al. ${ }^{48}$ reported a single lifetime of $(0.29 \pm 0.04)$ ps for adenosine in the same solvent. It is conceivable that the mono-exponential decay lifetime reported in this and other ${ }^{36,46}$ studies for AMP upon excitation at $267 \mathrm{~nm}$ is simply the weighted mean of the two exponential lifetimes determined Kwok et al. ${ }^{47}$ Alternatively, the time-zero coherent signal from the water solvent upon excitation at $267 \mathrm{~nm}$ may contribute to the additional lifetime reported by these authors. 

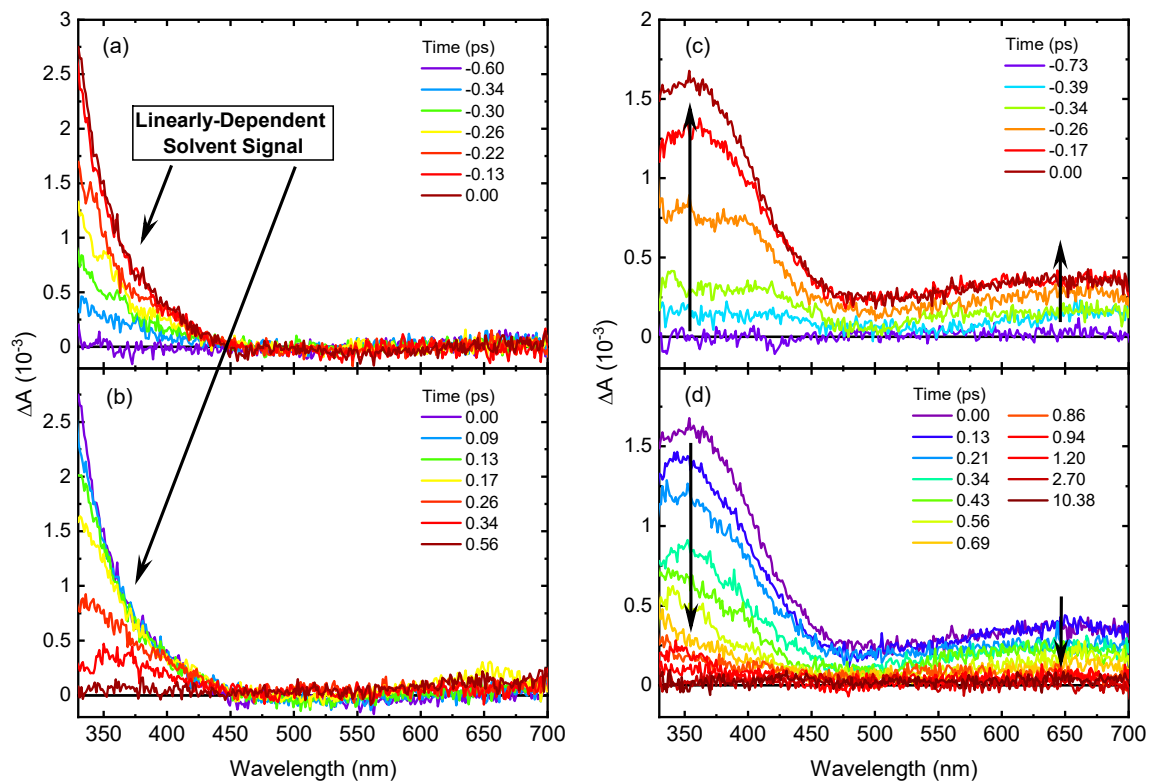

Figure 8. Spectral evolution of the transient absorption spectra of neat buffer (a-b) and adenosine 5'-monophosphate (AMP, c-d) in phosphate buffer $\mathrm{pH} 6.8$ following excitation at $267 \mathrm{~nm}$.
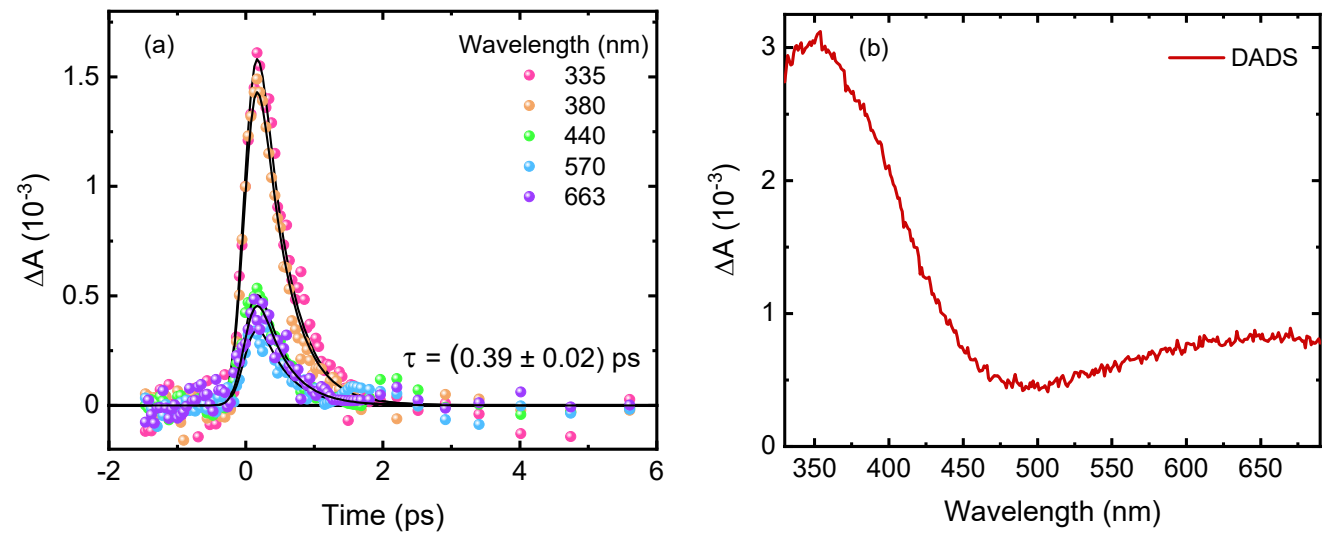

Figure 9. (a) Representative kinetic decay traces of AMP at select probe wavelengths globally fitted with a mono-exponential kinetic model. (b) Decay associated difference spectra (DADS) of AMP globally fitted with a mono-exponential kinetic model.

As shown in Figure 8, the experimental conditions used in this work eliminate the detection of the two-photon ionization of water in the phosphate buffer, but a time-zero coherent solvent signal is still observed in the spectral range from 320 to $450 \mathrm{~nm}$ (Figure 8a,b). As shown in Figure 10 , the amplitude of this signal increases linearly with the laser pump power and is independent of the intensity of the probe beam. Rasmusson et al. assigned this to a two-photon absorption (TPA) 
process where the strong pump field induces simultaneous absorption of one pump and one probe photon $\left(h v\right.$ pump $+h v$ probe) ${ }^{49}$

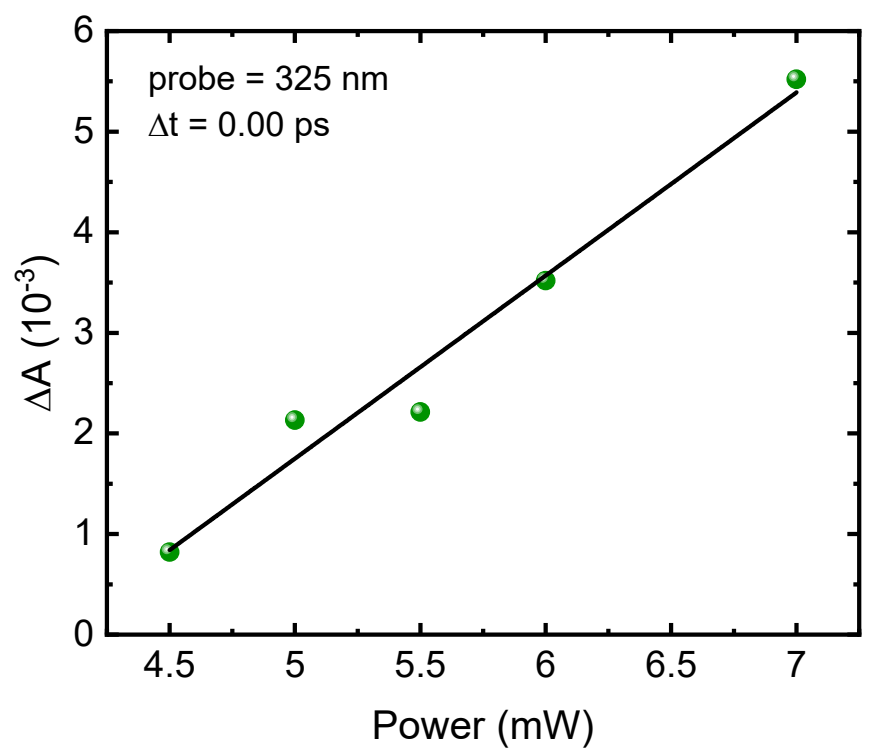

Figure 10. Dependence of the phosphate buffer signal at the probe wavelength of $325 \mathrm{~nm}$ with the average laser pump power at $267 \mathrm{~nm}$ before the optical chopper.

To determine whether a correction for the TPA coherent signal of the solvent was necessary under the experimental conditions used, an investigation was carried out by scaling the TPA coherent signal of the buffer-only to $5 \%$ (i.e., equivalent to the fraction of photons absorbed by the solvent in the GMP and 7dza solutions), and then subtracting the TPA coherent solvent signal from the transient absorption data of 7dza and GMP. This correction was observed to result in a negligible change in the transient absorption spectra of both 7dza and GMP (i.e., decreasing the $\Delta \mathrm{A}$ amplitude of the transient absorption spectra by less than $1 \%$ in the spectral probe region below $450 \mathrm{~nm}$ and significantly less in the spectral region from 450 to $700 \mathrm{~nm}$ ). 


\section{Supplementary Material}

DFT-optimized geometries and TD-DFT vertical excitation energies and Kohn-Sham orbitals for each conformation of 7dza and GMP; estimation of the population reaching the $\mathrm{L}_{\mathrm{a}}$ and Lb states upon excitation of 7dza and GMP at $267 \mathrm{~nm}$; normalized decay traces at different probe wavelengths; equations and procedures used to estimate the photon flux at $267 \mathrm{~nm}$.

\section{Dedication}

This paper is dedicated to Distinguished University Professor Mary D. Barkley, a superb biophysical chemist who developed and applied innovative approaches to investigate protein and DNA structure and interactions. She is an influential mentor and advocate for women and underrepresented groups in the sciences. Barkley was first woman professor in the Department of Chemistry and the first woman to hold an endowed chair in the natural sciences at CWRU.

\section{Acknowledgements}

The authors acknowledge the National Science Foundation (Grant No. CHE-1800052). This work made use of the High-Performance Computing Resource in the Core Facility for Advanced Research Computing at CWRU.

\section{Data Availability}

The data that support the findings of this study are available from the corresponding author upon reasonable request. 


\section{References}

${ }^{1}$ C.E. Crespo-Hernández, B. Cohen, P.M. Hare, and B. Kohler, Chem. Rev. 104, 1977 (2004).

${ }^{2}$ C.T. Middleton, K. de La Harpe, C. Su, Y.K. Law, C.E. Crespo-Hernández, and B. Kohler, Annu. Rev. Phys. Chem. 60, 217 (2009).

${ }^{3}$ A.A. Beckstead, Y. Zhang, M.S. De Vries, and B. Kohler, Phys. Chem. Chem. Phys. 18, 24228 (2016).

${ }^{4}$ C. Reichardt, C. Wen, R.A. Vogt, and C.E. Crespo-Hernández, Photochem. Photobiol. Sci. 12, 1341 (2013).

${ }^{5}$ C.E. Crespo-Hernández, L. Martínez-Fernández, C. Rauer, C. Reichardt, S. Mai, M. Pollum, P. Marquetand, L. González, and I. Corral, J. Am. Chem. Soc. 137, 4368 (2015).

${ }^{6}$ L. Martínez-Fernández, S. Arslancan, D. Ivashchenko, C.E. Crespo-Hernández, and I. Corral, Phys. Chem. Chem. Phys. 21, 13467 (2019).

${ }^{7}$ E.M. Arpa, M.M. Brister, S.J. Hoehn, C.E. Crespo-Hernández, and I. Corral, J. Phys. Chem. Lett. 11, 5156 (2020).

${ }^{8}$ S. Yamazaki, A.L. Sobolewski, and W. Domcke, Phys. Chem. Chem. Phys. 11, 10165 (2009).

${ }^{9}$ B. Cohen, P.M. Hare, and B. Kohler, J. Am. Chem. Soc. 125, 13594 (2003).

${ }^{10}$ K. Röttger, R. Siewertsen, and F. Temps, Chem. Phys. Lett. 536, 140 (2012).

${ }^{11}$ J.P. Villabona-Monsalve, R. Noria, S. Matsika, and J. Peón, J. Am. Chem. Soc. 134, 7820 (2012).

${ }^{12}$ M. Barbatti, A.J.A. Aquino, J.J. Szymczak, D. Nachtigallová, P. Hobza, and H. Lischka, Proc. 
Natl. Acad. Sci. U. S. A. 107, 21453 (2010).

${ }^{13}$ J. Chen and B. Kohler, Phys. Chem. Chem. Phys. 14, 10677 (2012).

${ }^{14}$ S. Matsika, Photoinduced Phenomena in Nucleic Acids I: Nucleobases in the Gas Phase and in Solvents; Eds. M. Barbatti, A. C. Borin, and S. Ullrich, Topics in Current Chemistry-Series; Springer-Verlag Berlin 335, 209 (2015).

${ }^{15}$ M. Pollum, L. Martínez-Fernández, and C. E. Crespo-Hernández, Photoinduced Phenomena in Nucleic Acids I: Nucleobases in the Gas Phase and in Solvents; Eds. M. Barbatti, A. C. Borin, and S. Ullrich, Topics in Current Chemistry-Series; Springer-Verlag Berlin 335, 245 (2015).

${ }^{16}$ F.D. Lewis, Photochem. Photobiol. 81, 65 (2005).

${ }^{17}$ N. Gorczak, T. Fujii, A.K. Mishra, A.J. Houtepen, F.C. Grozema, and F.D. Lewis, J. Phys. Chem. B 119, 7673 (2015).

${ }^{18}$ F.D. Lewis, P. Daublain, B. Cohen, J. Vura-Weis, and M.R. Wasielewski, Angew. Chemie Int. Ed. 47, 3798 (2008).

${ }^{19}$ F.D. Lewis, R.M. Young, and M.R. Wasielewski, Acc. Chem. Res. 51, 1746 (2018).

${ }^{20}$ S.O. Kelley and J.K. Barton, Chem. Biol. 5, 413 (1998).

${ }^{21}$ A.K. Thazhathveetil, M.A. Harris, R.M. Young, M.R. Wasielewski, and F.D. Lewis, J. Am. Chem. Soc. 139, 1730 (2017).

${ }^{22}$ F.D. Lewis, J. Liu, X. Liu, X. Zuo, R.T. Hayes, and M.R. Wasielewski, Angew. Chemie - Int. Ed. 41, 1026 (2002).

${ }^{23}$ F.D. Lewis, X. Liu, J. Liu, S.E. Miller, R.T. Hayes, and M.R. Wasielewski, Nature 406, 51 
(2000).

${ }^{24}$ V. Karunakaran, K. Kleinermanns, R. Improta, and S.A. Kovalenko, J. Am. Chem. Soc. 131, 5839 (2009).

${ }^{25}$ C.C.-W. Cheng, C. Ma, C.T.-L. Chan, K.Y.-F. Ho, and W.-M. Kwok, Photochem. Photobiol. Sci. 12, 1351 (2013).

${ }^{26}$ F.A. Miannay, T. Gustavsson, A. Banyasz, and D. Markovitsi, J. Phys. Chem. A 114, 3256 (2010).

${ }^{27}$ J. Lee, J.R. Challa, and D.W. McCamant, J. Phys. Chem. B 121, 4722 (2017).

${ }^{28}$ S.F. Altavilla, J. Segarra-Martí, A. Nenov, I. Conti, I. Rivalta, and M. Garavelli, Front. Chem. 3, 1 (2015).

${ }^{29}$ L. Serrano-Andrés, M. Merchán, and A.C. Borin, J. Am. Chem. Soc. 130, 2473 (2008).

${ }^{30}$ R. Krishnamurthy, Acc. Chem. Res. 45, 2035 (2012).

${ }^{31}$ J.R. Platt, J. Chem. Phys. 17, 484 (1949).

${ }^{32}$ C. Adamo, G.E. Scuseria, and V. Barone, J. Chem. Phys. 111, 2889 (1999).

${ }^{33}$ D. Tuna, A.L. Sobolewski, and W. Domcke, J. Phys. Chem. A 118, 122 (2014).

${ }^{34}$ D. Tuna and W. Domcke, Phys. Chem. Chem. Phys. 18, 947 (2015).

${ }^{35}$ J.-M.L. Pecourt, J. Peon, and B. Kohler, J. Am. Chem. Soc. 122, 9348 (2000).

${ }^{36}$ C.E. Crespo-Hernández and B. Kohler, J. Phys. Chem. B 108, 11182 (2004).

${ }^{37}$ M. J. Frisch, Gaussian 16, Revision A.01, Gaussian, Inc., Wallingford, CT, 2016. ${ }^{38}$ E. Cancès, 
B. Mennucci, and J. Tomasi, J. Chem. Phys. 107, 3032 (1997).

${ }^{39}$ V. Barone, M. Cossi, and J. Tomasi, J. Chem. Phys. 107, 3210 (1997).

${ }^{40}$ C. Reichardt, R.A. Vogt, and C.E. Crespo-Hernández, J. Chem. Phys. 131, 0 (2009).

${ }^{41}$ M. Pollum, S. Jockusch, and C.E. Crespo-Hernández, J. Am. Chem. Soc. 136, 17930 (2014).

${ }^{42}$ M.M. Brister and C.E. Crespo-Hernández, J. Phys. Chem. Lett. 10, 2156 (2019).

${ }^{43}$ J.J. Snellenburg, S. Laptenok, R. Seger, K.M. Mullen, and I.H.M. van Stokkum, J. Stat. Softw. 49, 1 (2012).

${ }^{44}$ P.M. Hare, E.A. Price, and D.M. Bartels, J. Phys. Chem. A 112, 6800 (2008).

${ }^{45}$ Y. Suzaki and A. Tachibana, Appl. Opt. 14, 2809 (1975).

${ }^{46}$ C.E. Crespo-Hernández, B. Cohen, and B. Kohler, Nature 436, 1141 (2005).

${ }^{47}$ W.-M. Kwok, C. Ma, and D.L. Phillips, J. Am. Chem. Soc. 128, 11894 (2006).

${ }^{48}$ J.M.L. Pecourt, J. Peon, and B. Kohler, J. Am. Chem. Soc. 123, 10370 (2001).

${ }^{49}$ M. Rasmusson, A.N. Tarnovsky, E. Åkesson, and V. Sundström, Chem. Phys. Lett. 335, 201 (2001). 